\title{
Non-hermitian oscillator-like Hamiltonians and $\lambda$-coherent states revisited
}

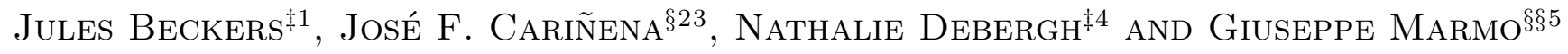 \\ ‡Theoretical and Mathematical Physics,Institute of Physics (B5), University of Liège, \\ B-4000 Liège 1 (Belgium) \\ ${ }^{\S}$ Departamento de Física Teórica, Universidad de Zaragoza, \\ 50009 Zaragoza, Spain.

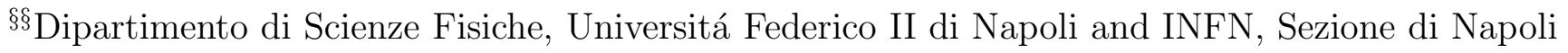 \\ Complesso Universitario di Monte Sant'Angelo, Via Cintia, 80125 Napoli, Italy
}

\begin{abstract}
Previous $\lambda$-deformed non-Hermitian Hamiltonians with respect to the usual scalar product of Hilbert spaces dealing with harmonic oscillator-like developments are (re)considered with respect to a new scalar product in order to take into account their property of selfadjointness. The corresponding deformed $\lambda$-states lead to new families of coherent states according to the DOCS, AOCS and MUCS points of view.
\end{abstract}

PACS: 03.65.Ca, 42.50.-p

\footnotetext{
${ }^{1}$ email: Jules.Beckers@ulg.ac.be

${ }^{2}$ email: jfc@posta.unizar.es

${ }^{3}$ Corresponding author

${ }^{4}$ Chercheur, Institut Interuniversitaire des Sciences Nucléaires, Bruxelles, email: Nathalie.Debergh@ulg.ac.be

${ }^{5}$ email: Giuseppe.Marmo@na.infn.it
} 
Coherence and squeezing have recently been revisited [1], 2] through new families of states depending on an extra $\lambda$-parameter. This real parameter has been introduced by exploiting the subnormal character [3] of the so-called (bosonic) creation operator acting on Fock spaces, a very well-known context inside harmonic oscillator-like developments.

A generalization of the factorization technique started by Schrödinger [4] was exploited in a recent paper [2] for analyzing some previous results on non-hermitian oscillator-like Hamiltonians with real spectra.

We recall (see e.g. [5] and references therein) that the classical factorization technique for a given Hamiltonian of a one-dimensional quantum system

$$
H=-\frac{1}{2} \frac{d^{2}}{d x^{2}}+V(x)
$$

consists in finding a constant $c$ and a superpotential function $W(x)$ such that

$$
H-c=A^{\dagger} A
$$

where

$$
A=\frac{1}{\sqrt{2}}\left(\frac{d}{d x}+W(x)\right) .
$$

In this case, the operator $A$ intertwines $H$ with the partner Hamiltonian $\widetilde{H}$ defined by $\widetilde{H}=A A^{\dagger}+c$, i.e., $A H=\widetilde{H} A$. Moreover, the adjoint operator $A^{\dagger}$ intertwines $\widetilde{H}$ with $H$, i.e., $A^{\dagger} \widetilde{H}=H A^{\dagger}$. In the particular case of the harmonic oscillator,

$$
H=-\frac{1}{2} \frac{d^{2}}{d x^{2}}+\frac{x^{2}}{2}
$$

the well-known annihilation and creation operators

$$
a=\frac{1}{\sqrt{2}}\left(\frac{d}{d x}+x\right), \quad a^{\dagger}=\frac{1}{\sqrt{2}}\left(-\frac{d}{d x}+x\right),
$$

play the role of $A$ and $A^{\dagger}$, while $c=\frac{1}{2}$. We recall that the operators $a$ and $a^{\dagger}$, together with the identity 1 , generate the Heisenberg algebra characterized by

$$
\left[a, a^{\dagger}\right]=1
$$

As it is well known, the commutation relations

$$
[H, a]=-a, \quad\left[H, a^{\dagger}\right]=a^{\dagger}
$$


can be used to obtain the full spectrum of the quantum harmonic oscillator operator. As $H>0$ there will be a state $\psi_{0}$ such that $a \psi_{0}=0$. Once it has been normalized it is given by

$$
\psi_{0}=\pi^{-1 / 4} e^{-\frac{1}{2} x^{2}},
$$

and the normalized eigenstates corresponding to the eigenvalues $E_{n}=\left(n+\frac{1}{2}\right)$, with $n=0,1, \ldots$, are

$$
\psi_{n}(x)=\frac{1}{\sqrt{n !}}\left(a^{\dagger}\right)^{n} \psi_{0}=\frac{\pi^{-\frac{1}{4}} 2^{-\frac{n}{2}}}{\sqrt{n !}} e^{-\frac{1}{2} x^{2}} H_{n}(x) .
$$

They form an orthonormal basis with respect to the standard inner product

$$
\left\langle\phi_{1}, \phi_{2}\right\rangle=\int_{-\infty}^{+\infty} \overline{\phi_{1}(x)} \phi_{2}(x) d x
$$

according to

$$
\int_{-\infty}^{+\infty} e^{-x^{2}} H_{n}(x) H_{m}(x) d x=\sqrt{\pi} 2^{n} n ! \delta_{n m}
$$

$H_{n}(x)$ being the very well-known Hermite polynomials [6]. In that context $x$ and $p=-i \frac{d}{d x}$ are self-adjoint operators associated with the important physical observables position and momentum, respectively.

Now, given a minimal numerable set of elements $\left\{\varphi_{0}, \varphi_{1}, \ldots, \varphi_{n}, \ldots\right\}$ of a separable Hilbert space $(\mathcal{H},(\cdot, \cdot))$ whose closure is $\mathcal{H}$, we can introduce a new inner product in $\mathcal{H}$ by means of a bilinear form \langle $\langle\cdot\rangle$,$\rangle such that$

$$
\left\langle\left\langle\varphi_{n}, \varphi_{m}\right\rangle\right\rangle=\delta_{m n}
$$

and a pair of operators $A$ and $A^{+}$such that

$$
\begin{aligned}
& A \varphi_{0}=0, \\
& A \varphi_{n}=\sqrt{n} \varphi_{n-1}, \\
& A^{+} \varphi_{n-1}=\sqrt{n} \varphi_{n} .
\end{aligned}
$$

Consequently, the operators $A$ and $A^{+}$so defined satisfy the Heisenberg algebra relation

$$
\left[A, A^{+}\right]=1 \text {. }
$$


Consider the Hamiltonian $H$ defined by

$$
H=\frac{1}{2}\left\{A, A^{+}\right\}=\frac{1}{2}\left(A A^{+}+A^{+} A\right)=A^{+} A+\frac{1}{2} .
$$

If we use it to define equations of motion we get the dynamics of the harmonic oscillator.

Indeed, as it was done in [7], 8] for nonlinear coherent states, we can define a map $D: \mathbb{C} \rightarrow \mathrm{Op}(\mathcal{H})$ as follows:

$$
D(z)=e^{\left(z A^{+}-\bar{z} A\right)}
$$

which defines a displacement operator. For any complex number $z$, let us define the state vectors $|z\rangle$ by

$$
|z\rangle=D(z) \varphi_{0}
$$

This is but a Weyl system in the Bargmann-Fock representation. The commutation rules of $A, A^{+}$and $H$ being he same ones as for the standard one-dimensional harmonic oscillator, the dynamical evolution will preserve the set of such vectors $|z\rangle$ and the dynamics associated with $H$ on $A$ and $A^{+}$ will define a dynamics on $\mathbb{C}$ by requiring it to be equivariant with respect to the $D$-map. Indeed, given a $z_{0}$ at $t=0$, the state evolves because $A$ and $A^{+}$ depends on $t$ and for any time $t$ we can define

$$
\left|z_{t}\right\rangle=e^{\left(z_{t} A^{+}-\bar{z}_{t} A\right)} \varphi_{0}
$$

giving rise, by derivation with respect to $t$ to

$$
\begin{aligned}
& \frac{d z}{d t}=i z \\
& \frac{d \bar{z}}{d t}=-i \bar{z}
\end{aligned}
$$

We can now consider the case of not only one, but a family $A_{\lambda}^{+}$of operators. The more general case of a two-parameter family proposed in 9] is quite similar. As already previously discussed [1], let us introduce the creation operator in the following way

$$
A_{\lambda}^{+}=a^{\dagger}+\lambda I, \quad \lambda \in \mathbb{R}
$$


where $a^{\dagger}$ is the adjoint of the annihilation operator $a$ given by (5), together with $A_{\lambda}=a$, and define

$$
\phi_{n \lambda}=\frac{1}{\sqrt{n !}}\left(A_{\lambda}^{+}\right)^{n} \psi_{0}
$$

where $\psi_{0}$ is the standard ground-state for the harmonic oscillator given by (7). Notice that $\phi_{0 \lambda}=\psi_{0}$ and $A_{\lambda}^{+} \phi_{n \lambda}=\sqrt{n+1} \phi_{n+1 \lambda}$.

As we suggested before we introduce a new inner product such that

$$
h_{\lambda}\left(\phi_{n \lambda}, \phi_{m \lambda}\right)=\left\langle\left\langle\phi_{n \lambda}, \phi_{m \lambda}\right\rangle\right\rangle=\delta_{n m} .
$$

We can now compute the adjoint of $A_{\lambda}^{+}$with respect to the inner product $\langle\langle\cdot, \cdot\rangle\rangle$. Indeed it is given by the relation

$$
\left\langle\left\langle\phi_{n \lambda},\left(A_{\lambda}^{+}\right)^{\dagger} \phi_{m \lambda}\right\rangle\right\rangle=\left\langle\left\langle A_{\lambda}^{+} \phi_{n \lambda}, \phi_{m \lambda}\right\rangle\right\rangle
$$

and therefore

$$
\sqrt{n+1} \delta_{n+1, m}=\sqrt{m} \delta_{n, m-1}=\left\langle\left\langle\phi_{n \lambda},\left(A_{\lambda}^{+}\right)^{\dagger} \phi_{m \lambda}\right\rangle\right\rangle
$$

from which we see that the adjoint of $A_{\lambda}^{+}$with respect to $\langle\langle\cdot, \cdot\rangle\rangle$ is given by

$$
\left(A_{\lambda}^{+}\right)^{\dagger} \phi_{n \lambda}=\sqrt{n} \phi_{n-1 \lambda}
$$

i.e., $\left(A_{\lambda}^{+}\right)^{\dagger}=a$, for any value of the parameter $\lambda$.

As $a$ and $A_{\lambda}^{+}$satisfy commutation relations as in (14), we can consider a Hamiltonian like in (15),

$$
H_{\lambda}=A_{\lambda}^{+} a+\frac{1}{2}=H_{0}+\lambda a
$$

and these developments lead to a non-Hermitian oscillator-like Hamiltonian [1] but with a real spectrum and specific eigenfunctions of special interest for discussing and comparing coherent as well as squeezed states with respect to already known ones [10, 11]. This is a direct consequence of the commutation rule $\left[A_{\lambda}, A_{\lambda}^{+}\right]=1$ and then $\widetilde{H}_{\lambda}=H_{\lambda}+1$.

In fact, this non-Hermitian character was associated with the scalar product (9) of the current Hilbert spaces we are dealing with in the conventional 
approaches. However, the Hamiltonian is hermitian with respect to the alternative inner product $\langle\langle\cdot, \cdot\rangle\rangle$, because the conjugate of $A_{\lambda}^{+}$is $a$. The new product can be expressed by

$$
\left\langle\left\langle\varphi_{1}, \varphi_{2}\right\rangle\right\rangle=\int_{-\infty}^{+\infty} \overline{\varphi_{1}(x)} \varphi_{2}(x) \rho(\lambda, x) d x,
$$

and the condition for $a$ to be the adjoint of $A_{\lambda}^{+}$is

$$
\left\langle\left\langle\varphi_{1}, A_{\lambda}^{+} \varphi_{2}\right\rangle\right\rangle=\left\langle\left\langle a \varphi_{1}, \varphi_{2}\right\rangle\right\rangle
$$

i.e.,

$$
\frac{\partial \rho}{\partial x}+\sqrt{2} \lambda=0
$$

with general solution

$$
\rho(\lambda, x)=C(\lambda) \exp (-\sqrt{2} \lambda x) .
$$

The arbitrary function $C(\lambda)$ can be fixed by imposing that $\left\langle\left\langle\phi_{0}, \phi_{0}\right\rangle\right\rangle=1$, and we will obtain

$$
C(\lambda)=\exp \left(-\frac{\lambda^{2}}{2}\right)
$$

Mean values and information coming from Heisenberg relations are now easy to evaluate in the new $\lambda$-context. The following results come out by exploiting generalized Poisson integrals such as [12]

$$
\int_{0}^{+\infty} e^{-l x^{m}} x^{k} d x=\frac{1}{m} l^{-\frac{k+1}{m}} \Gamma\left(\frac{k+1}{m}\right), \quad l>0, m>0, k>-1 .
$$

We get

$$
\langle x\rangle_{\lambda}=-\frac{\lambda}{\sqrt{2}}, \quad\left\langle x^{2}\right\rangle_{\lambda}=n+\frac{1}{2}+\frac{\lambda^{2}}{2}, \quad(\Delta x)_{\lambda}^{2}=n+\frac{1}{2},
$$

and

$$
\langle p\rangle_{\lambda}=-i \frac{\lambda}{\sqrt{2}}, \quad\left\langle p^{2}\right\rangle_{\lambda}=n+\frac{1}{2}-\frac{\lambda^{2}}{2}, \quad(\Delta p)_{\lambda}^{2}=n+\frac{1}{2},
$$

showing that squeezing is not possible in this $\lambda$-context while coherence can be developed in the usual $n=0$-context [10, 13. Moreover it is clear from 
these results that if $x$ is still Hermitian with respect to the new scalar product (18), $p$ is not. More precisely, it can be verified from (18) that the Hermitian conjugate of $p$ is $p+i \sqrt{2} \lambda$ implying that if we want to restore the Hermiticity for the momentum operator, we have to consider

$$
p_{\lambda} \equiv-i \frac{d}{d x}+i \frac{\lambda}{\sqrt{2}},
$$

instead of $p$. We then have

$$
\left\langle p_{\lambda}\right\rangle_{\lambda}=0, \quad\left\langle p_{\lambda}^{2}\right\rangle_{\lambda}=n+\frac{1}{2}, \quad\left(\Delta p_{\lambda}\right)_{\lambda}^{2}=n+\frac{1}{2}
$$

Let us now exploit the previous results and turn to the construction of new coherent states depending on $\lambda$. Following the DOCS (displacement operator coherent spates) point of view [13], it is straightforward to construct such states in terms of the new creation $\left(A_{\lambda}^{+}\right)$and annihilation $(a)$ operators through the displacement operator

$$
D_{\lambda}(z)=e^{\left(-\bar{z} a+z A_{\lambda}^{+}\right)},
$$

which allows us to introduce new coherent states,

$$
|z, \lambda\rangle=D_{\lambda}(z) \psi_{0}
$$

whose coordinate representation will be

$$
\psi_{z}(\lambda, x)=e^{z A_{\lambda}^{\dagger}-\bar{z} a} \psi_{0}(x)=e^{-\frac{1}{2}|z|^{2}} \sum_{n=0}^{\infty} \frac{z^{n}}{\sqrt{n !}} \phi_{n \lambda}(x)
$$

which are such that

$$
a \psi_{z}(\lambda, x)=z \psi_{z}(\lambda, x)
$$

ensuring also the AOCS (annihilation operator coherent spates) point of view. Due to the fact that the normalization of these states with respect to the scalar product (18)

$$
\int_{-\infty}^{+\infty} \overline{\psi_{z}(\lambda, x)} \psi_{z}(\lambda, x) \rho(\lambda, x) d x=1
$$


is realized, we have the following mean values

$$
\begin{gathered}
\langle x\rangle_{z}=\frac{1}{\sqrt{2}}(z+\bar{z}-\lambda), \\
\left\langle p_{\lambda}\right\rangle_{z}=\frac{i}{\sqrt{2}}(-z+\bar{z}),
\end{gathered}
$$

and similar results concerning $<x^{2}>_{z},<p_{\lambda}^{2}>_{z}$ finally leading to

$$
(\Delta x)_{z}^{2}=\left(\Delta p_{\lambda}\right)_{z}^{2}=\frac{1}{2}
$$

The third and last point of view, the MUCS (minimal uncertainty coherent states) one, is thus also ensured in this context of new $\lambda$-coherent states.

Coming back to the classical interpretation, the configuration space of the system we are considering is the complex plane $\mathbb{C}$ that can be identified with $\mathbb{R}^{2}$ according to

$$
z=\frac{1}{\sqrt{2}}(q+i p), \quad \bar{z}=\frac{1}{\sqrt{2}}(q-i p) .
$$

In this way we are choosing one of the possible complex structures in $\mathbb{R}^{2}$. We can define the inner product in $\mathbb{C}$ given by $h(z, w)=\bar{z} w$, whose real part provides one half of the standard Euclidean structure in $\mathbb{R}^{2}$, while the imaginary part gives us one half of the standard symplectic structure. The complex structure in $\mathbb{R}^{2}$ is defined by multiplication by $i$, i.e., $J z=i z$, then $J(q, p)=(-p, q)$. The tangent space to $\mathbb{R}^{2}$ at each point $m \in \mathbb{R}^{2}$ is identified in a natural way to $\mathbb{R}^{2}$ itself as a linear space. A local basis of the module of vector fields in $\mathbb{R}^{2}$ is made up by $\partial / \partial q$ and $\partial / \partial p$, and that of the differential forms by $d q$ and $d p$. The complexified of this space is also generated by

$$
d z=\frac{1}{\sqrt{2}}(d q+i d p), \quad d \bar{z}=\frac{1}{\sqrt{2}}(d q-i d p)
$$

and similarly, for the dual basis of vector fields,

$$
\frac{\partial}{\partial z}=\frac{1}{\sqrt{2}}\left(\frac{\partial}{\partial q}-i \frac{\partial}{\partial p}\right), \quad \frac{\partial}{\partial \bar{z}}=\frac{1}{\sqrt{2}}\left(\frac{\partial}{\partial q}+i \frac{\partial}{\partial p}\right) .
$$

In these complex coordinates, the canonical symplectic form is

$$
\omega=i d z \wedge d \bar{z} .
$$


Now, when comparing (5) with (34) we see that (16) can be seen in the classical approach as a transformation

$$
z \mapsto z_{\lambda}=z, \quad \bar{z} \mapsto \bar{z}_{\lambda}=\bar{z}+\lambda,
$$

which is a canonical transformation, but it does not preserve the Hamiltonian for the harmonic oscillator $H(z, \bar{z})=h(z, \bar{z})$, which transforms into

$$
H_{\lambda}=H+\lambda z \text {. }
$$

Therefore the dynamics

$$
\Gamma=i\left(z \frac{\partial}{\partial z}-\bar{z} \frac{\partial}{\partial \bar{z}}\right)
$$

changes to

$$
\Gamma_{\lambda}=\Gamma-i \lambda \frac{\partial}{\partial \bar{z}}
$$

However, the transformation being canonical, the image of closed orbits for the harmonic oscillator still remain closed, and moreover, the period is also constant as for the harmonic oscillator, therefore the quantum spectrum is equally spaced.

In summary, in the new variables the system is indeed an Harmonic Oscillator (both at the classical level and at the quantum level). However from the physical point of view, when we compare them in the same coordinate system, we find that they identify different physical systems because the equilibria points are different (at the classical level) and the vacua states are different at the quantum level. Even though they are represented abstractly by the algebra of the Harmonic Oscillator, their realizations identify different physical oscillators characterized by different zero modes. Thus the dynamics in the lambda variables has the same form as the other dynamics in the original coordinates. To compare them however, we need to express both in the same coordinate system and when this is done they are associated with different vector fields.

\section{References}

[1] J. Beckers, N. Debergh and F.H. Szafraniec, Phys. Lett. A 243 (1998) 256-60, Phys. Lett. A 246 (1998) 561-561. 
[2] N. Debergh, J. Beckers and F.H. Szafraniec, Phys. Lett. A 267 (2000) 113-16.

[3] F.H. Szafraniec, Comm. Math. Phys. 210 (2000) 323-34 and references therein.

[4] E. Schrödinger E., Proc. Roy. Irish Acad. A 46 (1940) 9-16; Proc. Roy. Irish Acad. A 46 (1940) 183-206; Proc. Roy. Irish Acad. A 47 (1941) 53-54.

[5] J. F. Cariñena, G. Marmo, A. M. Perelomov and M. F. Rañada: Int. J. Mod. Phys. A 13 (1998) 4913-29.

[6] W. Magnus, F. Oberhettinger, R.P. Soni, Formulas and Theorems for the Special Functions of Mathematical Physics (Springer, Berlin, 3rd Edition, 1966).

[7] P. Aniello, V. Man'ko, G. Marmo, S. Solimeno and F. Zaccaria, J. Opt. B:Quantum Semiclass. Opt. 2, (2000) 718-25.

[8] V. Man'ko, G. Marmo, A. Porzio, S. Solimeno and F. Zaccaria, Phys. Rev. A 62, 053407 (2000).

[9] X. Wang, L.C. Kwek and C.H. Oh, Phys. Lett. A 259 (1999) $7-14$.

[10] J.R. Klauder and B.S. Skagerstam, Coherent States, Applications in Physics and Mathematical Physics (World Scientific, Singapore, 1985).

[11] H.P. Yuen, Phys. Rev. A 13 (1976) 2226.

[12] W. Grobner and N. Hofreiter, Integraltafel, Zweiter Teil, Bestimmte Integrale (Springer, Wien, 1958).

[13] A.M. Perelomov, Generalized Coherent States and their Applications (Springer, New York, 1986). 